\title{
Optimum Design Considerations for a 3-DOF Micro Accelerometer Using Nanoscale Piezoresistors
}

\author{
Tan D. Tran ${ }^{1}$, Dzung V. Dao ${ }^{3}$, Tung T. Bui ${ }^{3}$, Long T. Nguyen ${ }^{1}$, Thuy P. Nguyen ${ }^{1,2}$, Sugiyama Susumu ${ }^{3}$ \\ ${ }^{l}$ MEMS and Microsystems Department, College of Technology, VNUH, VIETNAM \\ ${ }^{2}$ International Training Institute for Materials Science (ITIMS), HUT, VIETNAM \\ ${ }^{3}$ Research Organization for Science and Engineering, Ritsumeikan University, JAPAN
}

\begin{abstract}
This paper presents the design optimization of high performance three-degree of freedom silicon accelerometer. The purpose of this optimization is to achieve the high sensitivity and high resolution. The optimization has been performed based on considerations of junction depth, the doping concentration of the piezoresistor, the temperature coefficient sensitivity, the noise, and the power consumption. Taking advantage of high piezoresistive effect in nanoscale piezoresistor, the cross-sectional area of the piezoresistor is fabricated to be $15 \times 10^{4} \mathrm{~nm}^{2}$. The result shows that the sensitivity of the optimized accelerometer is improved while the resolution is comparable to previous results. The dimension of sensor is as small as $1 \mathbf{~ m m}^{2}$, so it is suitable for many immerging applications.
\end{abstract}

Keywords-optimization; nanoscale piezoresistor; accelerometter

\section{INTRODUCTION}

Recently, accelerometers are in great demand for many applications ranging from guidance and stabilization of the spacecrafts to research on vibrations of Parkinson patients' fingers. Generally, it is desirable that accelerometers have high sensitivity and fine resolution. Among the several technological alternatives, silicon piezoresistive accelerometers are noteworthy since it is compatible with microelectronic batch fabrication technology [1], and therefore, the die size and cost are reduced. There is an extensive research on silicon piezoresistive accelerometer to improve its performance and further miniaturization. However, a comprehensive analysis considering the impact of many parameters, such as the size effect, doping concentration and the temperature coefficient sensitivity of piezoresistor, noises, and power consumption on the sensitivity and resolution has not been reported.

This paper presents the full design and optimization of a 3DOF micro accelerometer utilizing nanoscale Si piezoresistors as sensing elements to enhance its sensitivity and resolution. The result is compared to previously-obtained experimental and theoretical data to show a considerable improvement of the optimized accelerometer.

\section{WORKING PRINCIPLE}

High sensitivity and small cross-axis sensitivity are important requirements for the 3 -DOF accelerometer. We proposed a configuration as shown in Fig. 1 to meet these critical characteristics. It consists of a proof mass connected to four surround beams, which are fixed to the outer frame at the centers.

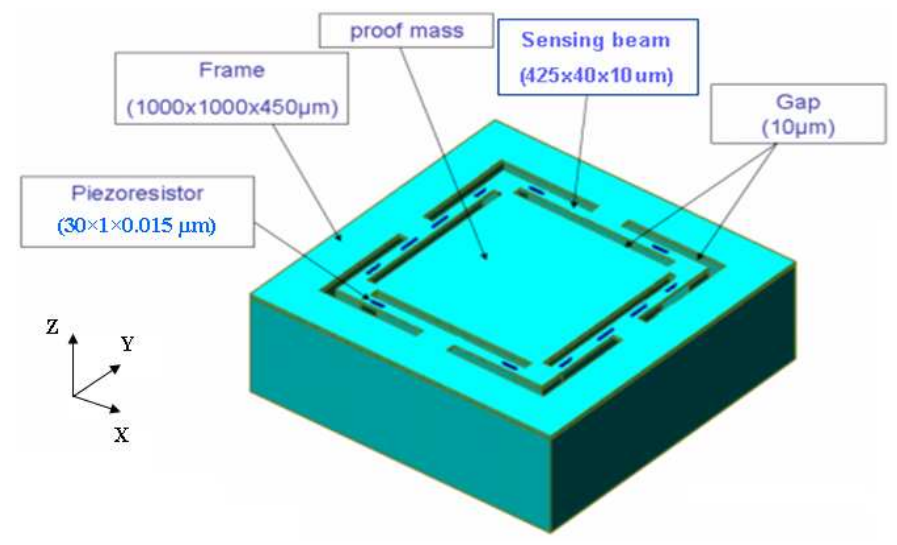

Figure 1. 3D model of the 3-DOF piezoresistive accelerometer

An external acceleration results in a force being exerted on the mass which results in a deflection of the proof mass. When a vertical acceleration (Az) applied to the sensor the mass will move up and down vertically. Similarly, when the $\mathrm{X}$ or $\mathrm{Y}$ component of transversal acceleration acting on the sensor, the mass will move laterally. The deflection of the proof mass causes stresses in four beams, resulting in resistance variation of the piezoresistors doped on the surface of the beam structure [2]. This variation is then converted into electrical signals by using three imbalance Wheatstone bridge circuits which are built by interconnecting twelve p-type piezoresistors. These piezoresistors are chosen to diffuse on the surface of these four beams because they can provide the maximal resistance variations and are aligned with the crystal directions $<110>$ and $<1 \overline{1} 0>$ of n-type silicon (100).

The piezoresistance effect is known to be caused by the anisotropic characteristics of the energy resolution in crystal space. In the silicon material, there are only three independent piezoresistive coefficients $\pi_{11}, \pi_{12}$ and $\pi_{44}$. The longitudinal piezoresistance coefficient $\pi_{l}$ is defined in the case the stress parallels with the direction of the electric filed and current density. Similarly, the transverse piezoresistance coefficient $\pi_{t}$ is defined in the case the stress is perpendicular with the direction of the electric filed and current density. In directions $<110>$ and $<1 \overline{1} 0>$ of n-type silicon (100), we can show these two coefficients thanks to three independent coefficients $\pi_{11}$, $\pi_{12}$ and $\pi_{44}$ as the following equation: 


$$
\begin{aligned}
& \pi_{l}=\frac{1}{2}\left(\pi_{11}+\pi_{12}+\pi_{44}\right) \\
& \pi_{t}=\frac{1}{2}\left(\pi_{11}+\pi_{12}-\pi_{44}\right)
\end{aligned}
$$

From simulation results in Fig. 3, we found that two normal stresses $\sigma_{2}$ and $\sigma_{3}$ are rather smaller when comparing to $\sigma_{1}$. This phenomenal will affect to the sensitivity of the sensor. To eliminate this effect, we should avoid placing piezoresistors near the fixed end and the start of the beam. Thus, we can calculate the relative change of resistance due to the normal stress by the following equation:

$$
\frac{\Delta R}{R}=\pi_{l} \sigma_{l}+\pi_{t} \sigma_{t}
$$

\section{DESIGN AND ANALYSIS UsING ANSYs}

Firstly, the structural analysis of the sensing chip was done by a modified nodal analysis (MNA) method for a quick estimation of sensor dimensions based on the required ranges of acceleration and the dimensions of the beam. Then, the finite element method (FEM) is applied to perform analyses of the stress distribution in the flexure beams. Based on the stress distribution, piezoresistors are placed to eliminate the crossaxis sensitivities and to maximize the sensitivities to three components of acceleration. The finite element model of the sensing chip was analyzed by using ANSYS software.

Fig. 2 shows the mesh generation for analysis and Fig. 3 shows the stress distribution in the longitudinal and transverse directions of the first beam caused by the acceleration Az. Clearly, the stress distribution in the direction along the beam (the longitudinal stress) is much larger than that of the transverse one.

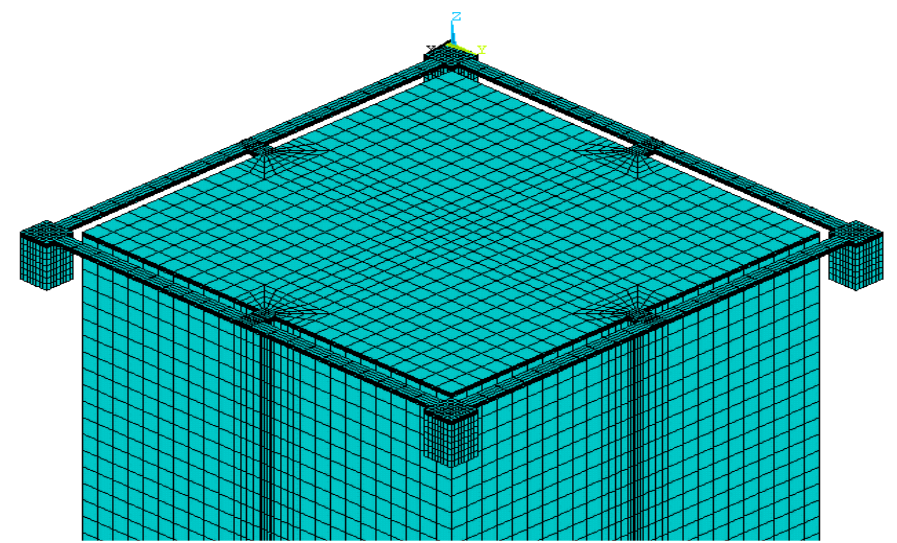

Figure 2. The dense mesh generation of the FEM model.

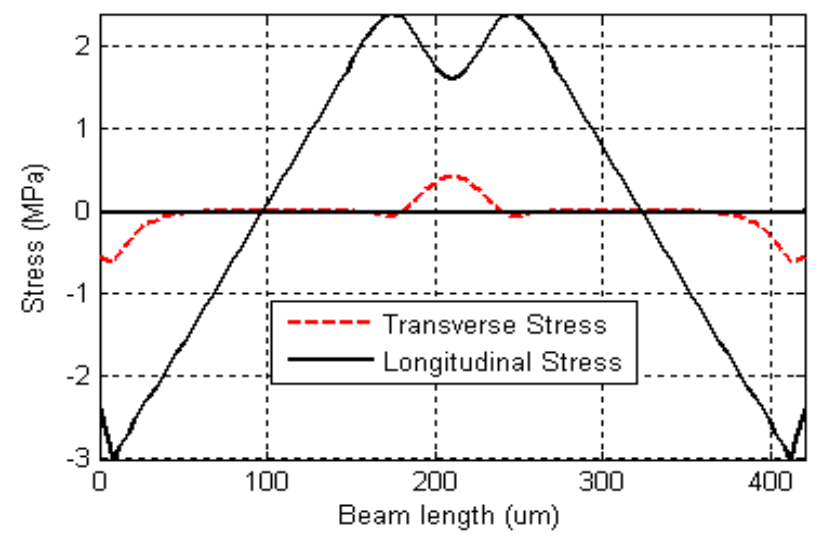

Figure 3. Stress distribution of a beam caused by vertical acceleration Az.

Based on this distribution, piezoresistors are positioned to eliminate the cross-axis sensitivities and to maximize the sensitivities to the three acceleration components. The behavior of the sensor is described in Table 1. To detect the accelerations, twelve identical p-type piezoresistors are shallowly diffused on the surface of the beams and interconnected to form three Wheatstone bridges in a manner like which was reported in Ref. [2].

TABLE I. RESISTANCE VALUES CHANGES WITH THREE COMPONENTS OF ACCELERATION

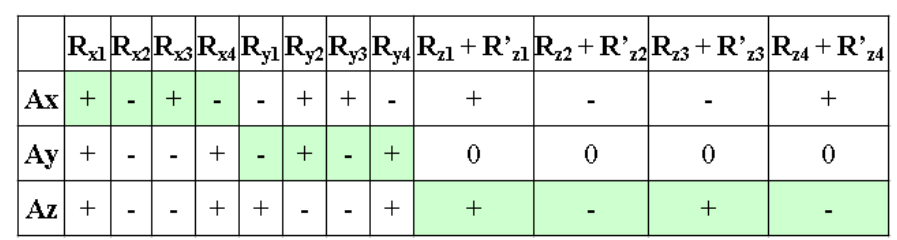

\section{OTIMUM DESIGN CONSIDERATION}

The mechanical sensitivities of each components of acceleration can be respectively expressed as:

$$
S_{\text {stress }}^{i}=\frac{\sigma^{i}}{a_{i}} \quad i=1,2,3
$$

Where $S_{\text {stress }}^{i}$ is the mechanical sensitivity and $\sigma^{i}$ is longitudinal stress induced by the acceleration $i^{\text {th }}$ component $a_{i}$. The electronics sensitivity can be given by:

$$
S_{i}=\frac{V_{\text {out }}}{a_{i}}=\frac{\Delta R}{R} V_{\text {in }}=\pi_{l} S_{\text {stress }}^{i} V_{\text {in }}
$$

Where $\mathrm{S}_{\mathrm{i}}$ and $\mathrm{V}_{\text {out }}$ are the sensitivity to the $\mathrm{i}^{\text {th }}$ acceleration component and output voltage, respectively.

The sensitivity depends not only on the structure and the positions of piezoresistors, but also on the length, the crosssectional area, and doping concentration of the piezoresistor. 
The cross-sectional area of piezoresistor is optimized to be $100 \mathrm{~nm} \times 1500 \mathrm{~nm}$ to increase the longitudinal piezoresistive coefficient and reduce the transverse one [3]. In general, temperature affects the piezoresistive coefficient through a change in the mobility and carrier concentration $(\mathrm{N})$ in the respective bands $[4,5]$. The dependence of the piezoresistive coefficient on the impurity concentration at a given temperature $(\mathrm{T})$ can be obtained by multiplying the piezoresistive factor $\mathrm{P}(\mathrm{N}, \mathrm{T})$ by the $\mathrm{PR}$ coefficient at room temperature $\left(\mathrm{T}_{0}\right)$ as follows:

$$
\pi(T)=\pi\left(T_{0}\right) P(N, T)
$$

Where

$$
P(N, T)=\frac{300}{T} \frac{F_{s+0.5}^{\prime}\left(E_{F} / K T\right)}{F_{s+0.5}\left(E_{F} / K T\right)}
$$

Where $\mathrm{K}$ is Boltzmann constant and $\mathrm{T}$ is temperature. The Fermi integral is the function of temperature and the Fermi energy $E_{F}$ [4]. The temperature strongly determines the value of $\pi(N, T)$. The higher it is, the smaller is the effect. Also larger concentration decreases the value of the piezoresistance coefficient.

The temperature of piezoresistor is decided by power consumption [6]. The steady state temperature rise of the selfheat piezoresistor $\left(T_{s s}\right)$ is shown as below:

$$
T_{s s}=\frac{P}{4 \pi d k}\left[1+\frac{2 k}{h R}\right] \approx \frac{P}{2 \pi d h R}
$$

Where $P$ is the power consumption of each piezoresistor, $d$ represents the thickness of the beam, $h$ represents the heat transfer coefficient, and $R$ represents the width of the beam respectively.

Fig. 4 shows the dependence of sensitivity on doping concentration and length of piezoresistor at specific power consumption. It is observed that the sensitivity decrease monotonically with the impurity concentration. The sensitivity also varies significantly with the beam length. It is noted that the stress distribution on the beam must be taken into account. The piezoresistive effect on each piezoresistor is determined through the average value of stress. However, changing the length of piezoresistor from $6 \mu \mathrm{m}$ to $30 \mu \mathrm{m}$ does not affect this average value of stress. We found that the optimum length of piezoresistors and doping concentration are of $30 \mu \mathrm{m}$ and $1 \times$ $10^{17}$ atoms $\mathrm{cm}^{-3}$, respectively. Concentration below $10^{17}$ atoms $\mathrm{cm}^{-3}$ is not practical due to instable Ohmic contact.

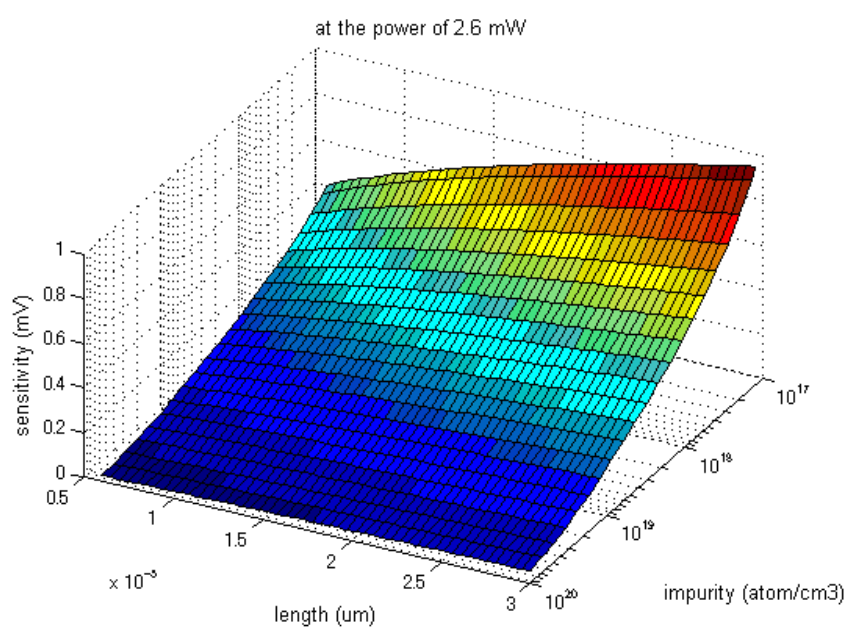

Figure 4. Variation of sensitivity with respect to different piezoresistor lengths and doping concentrations

Resolution is defined as the noise divided by the sensitivity. It is observed that optimization of the resolution has been achieved by increasing the sensitivity and reducing the noise. There are two typical noise sources existing in all piezoresistive sensors, including the Johnson noise and flicker noise [7]. The noises depend on the bandwidth of sensor, the temperature, the geometry of piezoresistor, the doping concentration and also the thickness of the beam. The variation of the resolution with specific power consumption for different doping concentrations and different piezoresistor lengths is shown in Fig. 5. Unlike the sensitivity, the optimum resolution is achieved with the $9 \times 10^{18}$ atoms $\mathrm{cm}^{-3}$. This is because the optimal values are achieved by the compromise among different parameters.

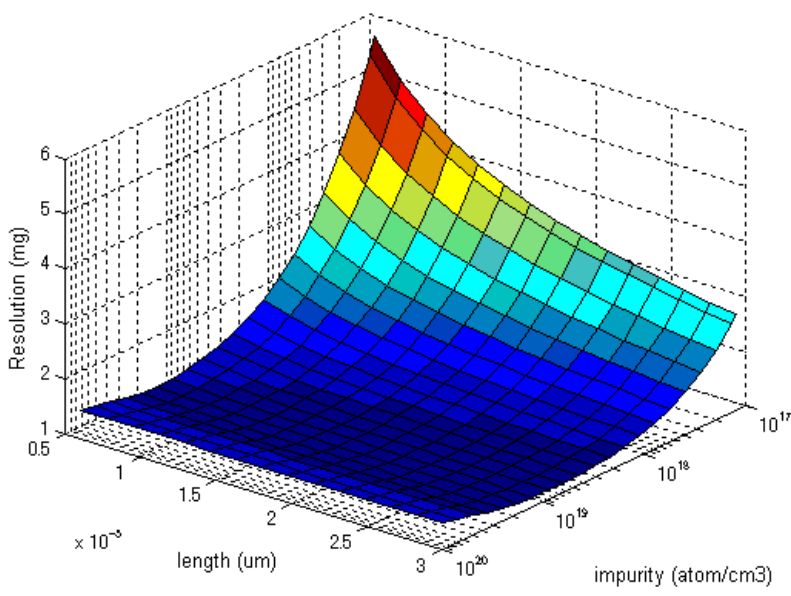

Figure 5. Variation of resolution with respect to different piezoresistor lengths and doping concentrations

Thus, a trade-off between high sensitivity and high resolution is necessary to decide the doping concentration in the range from $1 \times 10^{17}$ atoms $\mathrm{cm}^{-3}$ to $9 \times 10^{18}$ atoms $\mathrm{cm}^{-3}$. 
The sensing chip was fabricated by micromachining process. Starting material is a multi layers SOI wafer. Thermal diffusion is performed to form p-type $\mathrm{Si}$, EB lithography and RIE to create nanoscale piezoresistors, metallization process to make interconnection, and deep RIE to define the beam and proof mass. Fig. 6 shows respectively the photographs of fabricated chip and the sensor packaging. Fig. 7 also shows for comparison the expected result of our new design and the testing in the previous paper. Obviously, the new design gives much higher sensitivity. Work on the fabrication of this optimized accelerometer is in progress.

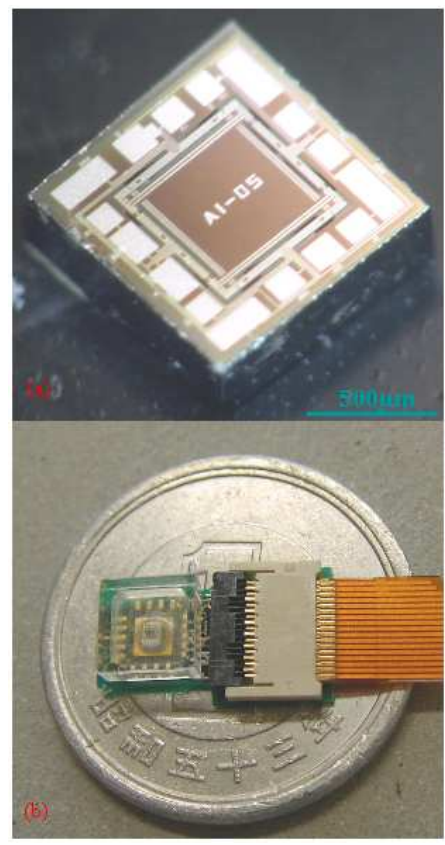

Figure 6. (a) Micrograph of a fabricated chip and (b) Sensor packaging for testing of our previous design

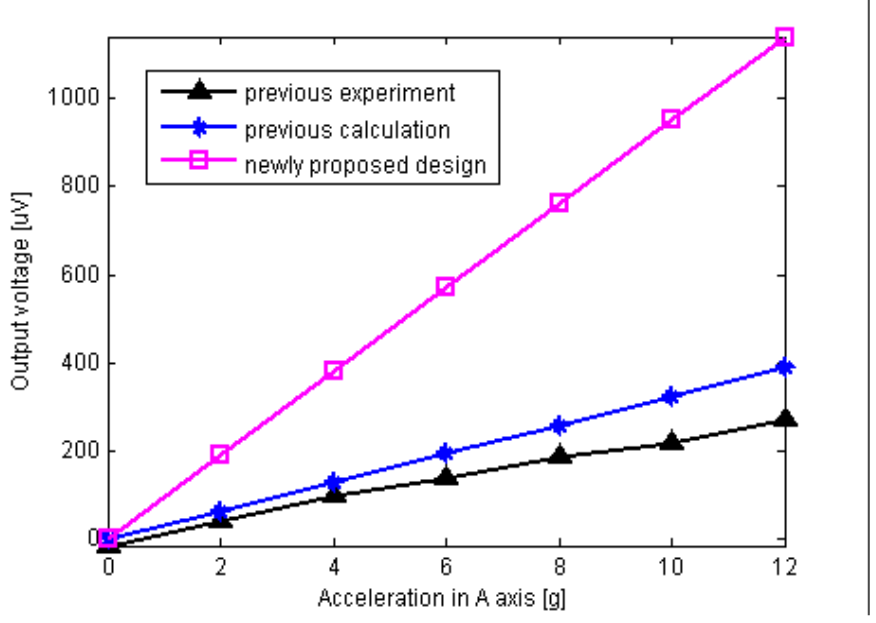

Figure 7. Comparisons among previous experiment, previous calculation, and our new proposed design

\section{CONCLUSION}

This paper presents a full design and analysis for a specific MEMS accelerometer. The piezoresistive effect was used as sensing principle of the sensor. The design results can be closest to fabrication condition by sensitivity error analysis. The miniature 3-DOF sensor is expected to be applied in some applications such as sign language recognition or patient monitoring system.

\section{ACKNOWLEDGMENT}

This work is supported from the Ministry of Science \& Technology of Vietnam, project No. 410506. The author also would like to thank for the support of Research Center for Nanomachine System Technology, Ritsumeikan University, Japan.

\section{REFERENCES}

[1] Yozo Kanda, "Piezoresistance Effect of Silicon", Sensors and Actuators, Vol. A 28, pp. 83-91, 1981.

[2] Dzung Viet Dao, Toshiyuki Toriyama, John Wells and Susumu Sugiyama, "Silicon Piezoresistive Six-Degree of Freedom ForceMoment Micro Sensor", Sensors and Materials, Vol. 15, No.3, pp. 113$135,2003$.

[3] Dzung Viet Dao, Toshiyuki Toriyama, and Susumu Sugiyama, "Noise and Frequency Analyses of a Miniaturized 3-DOF Accelerometer Utilizing Silicon Nanoscale Piezoresistors", in The 3rd Int'l Conference on Sensors, Vol. 3, pp. 1464-1467, 2004.

[4] Y. Kanda, "Graphical representation of the piezoresistance coefficients in Si shear coefficients in plane", Japanese J. Appl. Phys., vol. 26, No. 7, pp. 1031-1033, 1987.

[5] Sekalski, P. Pons, and P. Napieralski, "Finite elements simulations of piezoresistive sensor of blood pressure", in Proceedings of the 7th International Conference, pp. 509- 512, 2003.

[6] C.Pramanik, S.Banerjee, D.Mukherjee, and H.Saha, "Development of SPICE Compatible Thermal Model of Silicon MEMS Piezoresistive Pressure Sensor for CMOS- MEMS Integration", in IEEE SENSORS pp. $761-764,2006$.

[7] R. P. Spencer, B. M. Fleischer, P. W. Barth, and J. B. Angell, "A theoretical study of transducer noise in piezoresistive and capacitive pressure sensors", IEEE Trans. Electron Devices., vol. 35, pp. 1289$1298,1988$. 